\title{
Immunolocalization of Goi-3 in foetal lung fibroblasts
}

\author{
A. Dosanjh
}

\begin{abstract}
Immunolocalization of Goi-3 in foetal lung fibroblasts. A. Dosanjh. CERS Journals Ltd 1998.

ABSTRACT: Guanosine triphosphate (GTP)-binding proteins or G proteins are involved in a wide variety of well-recognized signalling activities between cell surface receptors and effectors. The heterotrimeric $\mathbf{G}$ proteins have $\alpha, \beta$ and $\gamma$ subunits organized in a trimeric structure. The aim of this study was to localize Goi-3, an important heterotrimeric $G$ protein, in foetal lung cells.

Using a foetal lung fibroblast cell line (RFL-6), the localization of Goi-3 was determined by immunofluorescence using a specific antibody to Gai-3, colocalization with a lectin known to bind the Golgi complex and Western blotting of RFL-6 cellular membrane proteins.

This study identified Goi-3 on the Golgi membranes in rat foetal lung cells. Treatment with cycloheximide, to block protein synthesis, diminished the cytosolic distribution of the protein, but intense Golgi staining remained. Goi-3, therefore, appears to be part of the Golgi complex and not present transiently during protein synthesis.

In the nonpolar foetal lung fibroblasts studied, the intracellular concentration of Goi-3 suggests a role for this protein in the intracellular trafficking and regulation of proteins needed for normal lung development.

Eur Respir J 1998; 12: 1137-1140.
\end{abstract}

Correspondence: A. Dosanjh

Stanford University School of Medicine

Stanford

California

USA

Fax: 16504987854

Keywords: Fibroblasts

foetal lung

$\mathrm{G}$ protein

Received: October 241997

Accepted after revision May 181998
The normal development of the lung is dependent on signal transduction and communication within and between cells. A major family of proteins known as guanine nucleotide regulatory proteins ( $\mathrm{G}$ proteins) is used in translating extracellular signals into intracellular events [1-3]. In particular, the high molecular weight $\mathrm{G}$ proteins, which are heterotrimers composed of $\alpha, \beta$ and $\gamma$ subunits, have been traditionally associated with transduction at the cell surface, rather than with intracellular activities, such as constitutive protein secretion [4]. These subunits have been conserved in eukaryotes throughout evolution. Functionally, $\mathrm{G}$ proteins can be classified as $\mathrm{G}_{\mathrm{s}}$, activated by cholera toxin, or $\mathrm{Gi}$, inhibited by pertussis toxin. The family of guanosine triphosphate (GTP)-binding proteins has been expanded to include proteins involved in secretion [5].

Low molecular weight $\mathrm{G}$ proteins, such as rab6p, have been associated with the Golgi complex and identified in the regulation of vesicular trafficking and constitutive protein secretion [6]. Heterotrimeric Goi-3 has also been found on the Golgi of polarized mature renal epithelial cells [7]. The topographic segregation of the Goi-3 subunit in foetal lung fibroblasts may suggest a functional intracellular role for the $\alpha$ subunit during lung development.

Pertussis toxin-sensitive Goi-3 could play an important role in the foetal lung by regulating Golgi trafficking and vesicular protein transport. The present study was conducted to determine the association of Goi-3 with Golgi complex membranes in rat foetal lung fibroblasts (RFL$6)$.
Materials and methods

\section{Cell culture}

RFL-6 (American Type Culture Collection, Rockville, MD, USA) is a cell line derived from the foetal lung of Sprague-Dawley rats. The cells were grown and maintained in Hams F12K medium (Gibco, Washington, DC, USA) containing $20 \%$ foetal bovine serum, $2 \%$ glutamine, $100 \mathrm{U} \cdot \mathrm{mL}^{-1}$ penicillin and $100 \mathrm{U} \cdot \mathrm{mL}^{-1}$ streptomycin at $37^{\circ} \mathrm{C}$ in $5 \% \mathrm{CO}_{2} / 95 \%$ air. After growth to an adherent monolayer, at days 3-5 after passage, when Goi-3 expression is maximal, the cells were treated with $0.1 \%$ trypsinethylenediaminetetraacetic acid (EDTA) in phosphatebuffer-ed saline (PBS) and detached. The medium containing serum was used to deactivate the trypsin. The cells were then grown on sterile glass coverslips and used after 3-5 days.

\section{EC antibody characterization}

The EC antibody (Dupont, Boston, MA, USA) binds with specificity to the C-terminus of the Gai-3 subunit. The term EC refers to the original clone of Goi-3. The EC antibody has some cross-reactivity with Go, which is not found in rat foetal lung cells [8]. The antibody is a polyclonal rabbit antibody and is supplied as rabbit antisera (New England Nuclear \& Dupont, Boston, MA, USA). It has been confirmed, by the supplier, to bind to the Goi-3 subunit at a 1:1,000 dilution in Western blotting. 


\section{Immunocytochemistry}

Cells were fixed with methanol:acetic acid (3:1), permeabilized with $0.1 \%$ Triton-X100 and then incubated sequentially with EC antibody and goat anti-rabbit immunoglobulin $\mathrm{G}$ (IgG)-fluorescein isothiocyanate (FITC) (Kirkegaard and Perry, Gaithersberg, MD, USA). Goi-3 and wheatgerm agglutinin (WGA)-rhodamine (Vector Laboratories, Burlinghame, CA, USA) were colocalized by double labelling the cells. The negative control staining was performed with nonimmune serum and another control was not treated with any primary antibody. WGA is a well-recognized marker of the Golgi complex [7]. Cells were viewed by conventional epifluorescence microscopy on a Nikon Microphot Microscope.

\section{Cycloheximide treatment}

Cells were pretreated with $10 \mu \mathrm{g} \cdot \mathrm{mL}^{-1}$ cycloheximide (Sigma, St Louis, MO, USA) and incubated at $37^{\circ} \mathrm{C}$ for 24 h. The cells were fixed and incubated with $\mathrm{EC}$ and goat antirabbit IgG-FITC under the same conditions as the control cells.

\section{Membrane preparation}

Adherent cells were rinsed in cold PBS and 3-5 mL homogenization buffer $(0.25 \mathrm{mM}$ sucrose, $10 \mathrm{mM}$ tris(hydroxymethyl) aminomethane (Tris), 1 mM EDTA, pH 7.4) was added to each dish. The cells were then scraped and homogenized. The nuclear pellet was discarded after the cells were centrifuged at $1,000 \times g$ for $10 \mathrm{~min}$ at $4^{\circ} \mathrm{C}$. The supernatant was then centrifuged at $100,000 \times g$ for $2 \mathrm{~h}$ at $4^{\circ} \mathrm{C}$. This supernatant (cytosol) and the pellet (membrane) were stored at $-80^{\circ} \mathrm{C}$ and a small aliquot from each was used for protein determination by the BioRad standard protein assay method (Hercules, CA, USA).

\section{Western blotting}

Aliquots of RFL-6 cell membrane and cytosolic proteins were solubilized by boiling in sample buffer $(1 \%$ sodium dodecyl sulphate (SDS), $30 \mathrm{mM}$ Tris, $\mathrm{pH}$ 6.8, $12 \%$ glycerol). After protein separation on a $10 \%$ SDS polyacrylamide gel, the proteins were then transferred to an Immobilon Membrane (Millipore Continental Water Systems, Bedford, MA, USA). The membrane was stained with Ponceau $\mathrm{S}$ in buffer to ensure equal transfer of the proteins. The destained membrane was then blocked in Superblock blotting buffer (Pierce, Rockford, IL, USA). Immunoblotting was performed with the EC antibody, to detect Goi-3, followed by alkaline phosphatase-conjugated secondary antirabbit IgG antibody. Developing solution was used according to the assay protocol (BioRad). Altern-atively, the membrane was processed in a high-sensitivity, nonradioactive chemiluminescent detection system (ECL; Amersham, Little Chalfont, UK). An autoradiograph of the immunoblot was then obtained.

\section{Results}

The Goi-3 subunit was localized by immunofluorescence staining with EC in the RFL-6 cell line. Goi-3 was shown to have a distinct pattern of distribution in the RFL-6 cell line. Intense fluorescence was present in a perinuclear crescent-shaped distribution, which is characteristic of Golgi complex staining (fig. 1). The negative control cells showed no such staining pattern. When the cells were sequentially treated with WGA, the Goi-3 staining colocalized to the Golgi. WGA has been used previously as a marker for the Golgi complex [7]. The WGA staining was more pronounced in the cytoplasm than was Goi-3-specific staining. The perinuclear colocalization corresponds to the Golgi complex in cells treated with both EC and WGA. WGA preferentially binds $N$-acetylglucosamine and sialic acid moieties and is thus more widely distributed intracellularly than Goi-3 (fig. 2). When RFL-6 cells were treated with cycloheximide to stop protein synthesis, there was persistent intense perinuclear staining of the Golgi comp-lex with EC antibody. The identification of Golgi staining specific for Goi-3 supports the finding that Goi-3 is a resident Golgi protein, rather than a transient protein being synthesized and processed in the Golgi complex (fig. 3). All immunocytochemistry was repeated in four separate experiments. Western blotting with EC antibody confirm-ed the presence of the Goi-3 $41 \mathrm{kDa}$ subunit on the membranes of RFL-6 cells (fig. 4). The band corresponding to Goi-3 was readily detectable and this result was confirmed in three separate experiments, by both alkaline phospha-tase conjugation and ECL methods.

\section{Discussion}

The present study establishes for the first time the location of Goi-3, a pertussis toxin-sensitive heterotrimeric $\mathrm{G}$ protein subunit, on the intracellular membranes of cultured rat foetal lung cells and its topographic concentration implies a role in Golgi trafficking and regulation of

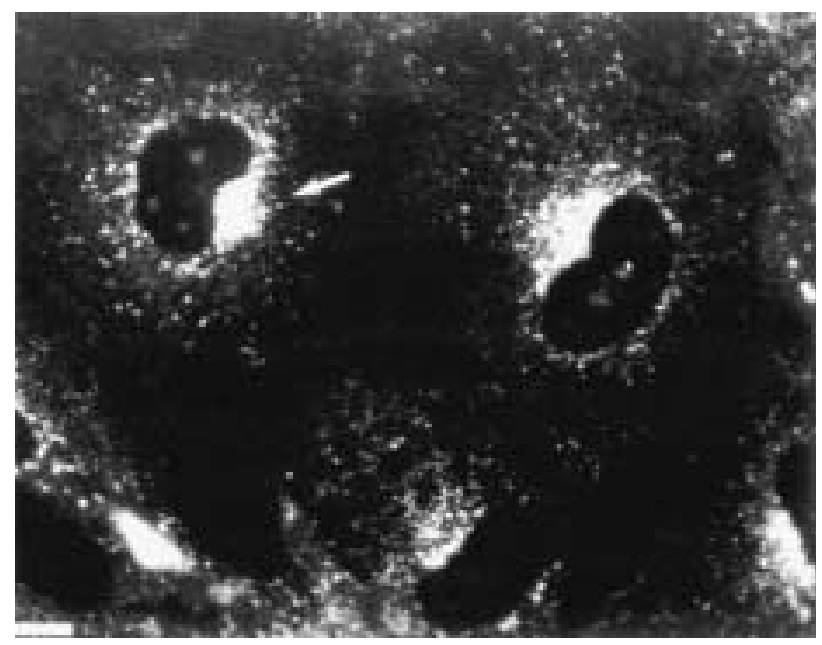

Fig. 1. - Immunocytochemical localization of Goi-3 in RFL-6 cells. The cells were incubated with anti-Goi-3 peptide antibody followed by a secondary antibody, goat anti-rabbit immunoglobulin $\mathrm{G}$ conjugated to fluorescein isothiocyanate. The areas of intense fluorescence are perinuclear and compact (arrow). This is an immunofluorescence pattern characteristic of Golgi complexes. (Internal scale bar $=10 \mu \mathrm{m}$.) 

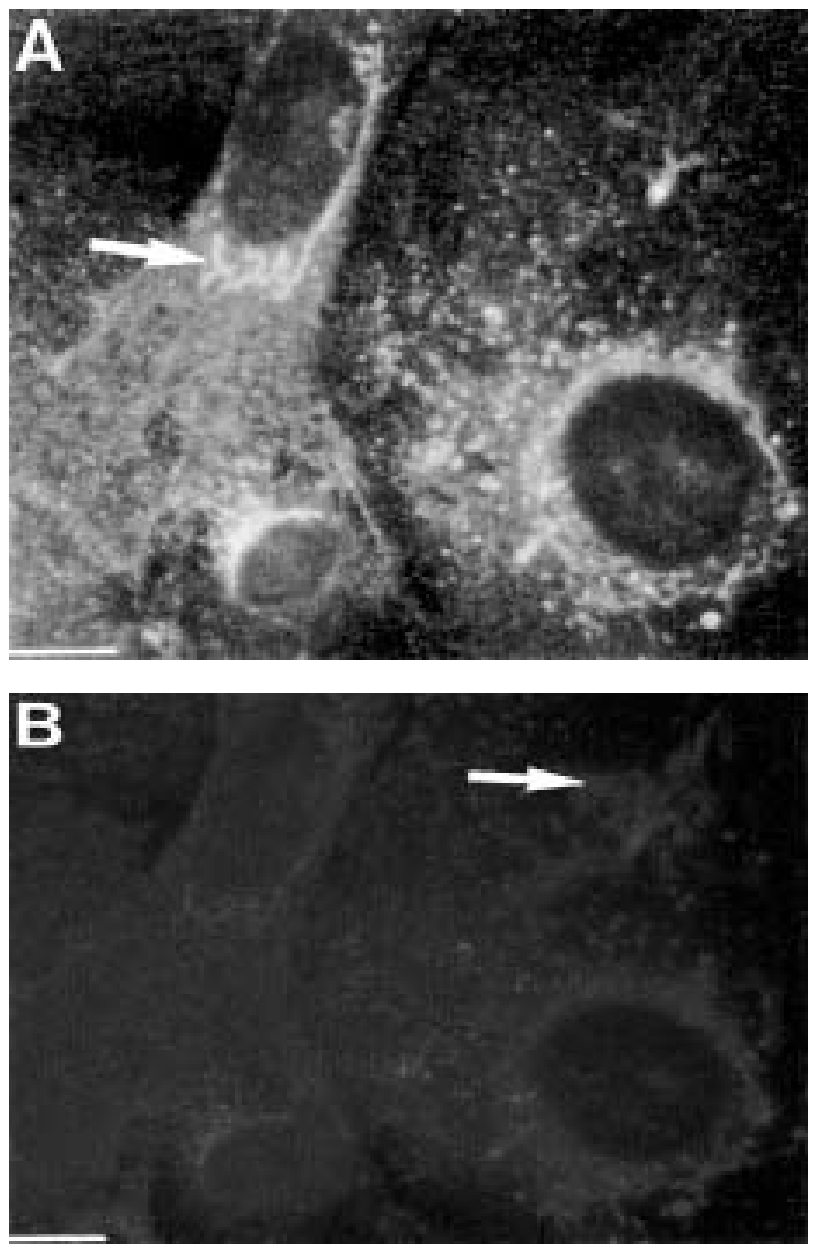

Fig. 2. - Colocalization of Gai-3 and wheatgerm agglutinin to the Golgi complex of cultured foetal rat lung cells. A) RFL-6 cells were incubated with anti-Gai-3 antibody followed by a secondary antibody, goat anti-rabbit immunoglobulin conjugated to fluorescein isothiocyanate. Intense perinuclear staining (arrow) is demonstrated, as well as punctate cytoplasmic staining. B) The same cells were then sequentially incubated with wheatgerm agglutinin conjugated to rhodamine. The lectin staining is both perinuclear and cytoplasmic. The pattern of perinuclear Golgi complex staining is colocalized with Goi-3. The arrow in B) shows an area that is not associated with the Golgi and is not colocalized. (Internal scale bar=10 $\mu \mathrm{m}$.)

constitutive protein secretion in the developing lung. The close association between protein intracellular localization and function has been reported previously [9-11]. While this study did not determine the precise various potential functions of Goi-3, its intracellular localization has implications for such future studies.

In RFL- 6 cells, Goi- 3 was predominantly present on the Golgi complex and appeared less prominently in a punctate diffuse cytoplasmic distribution. Cycloheximide-treated cells also have the characteristic perinuclear crescentshaped staining pattern, indicating that Goi-3 is not merely transiently present in the Golgi complex, but rather is part of the complex itself. Colocalization of Goi-3 with an already established marker for the Golgi complex provides further evidence that the immunolocalization of the protein is consistent with a Golgi complex distribution.

The Goi-3 subunit was also identified by Western blotting of the RFL- 6 membrane proteins. The $41 \mathrm{kDa}$ pro-
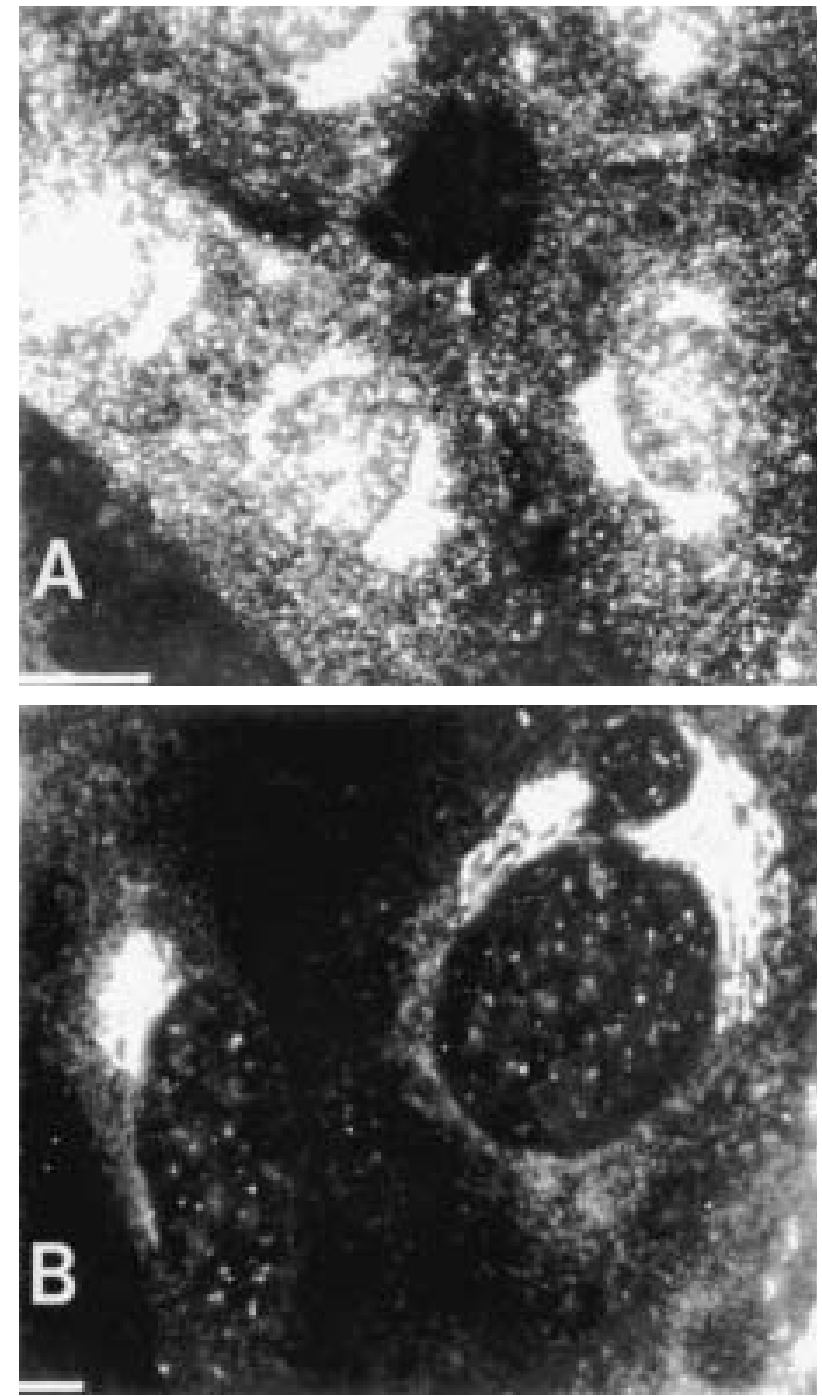

Fig. 3. - Effect of cycloheximide on Goi-3 localization. A) Perinuclear and cytoplasmic fluorescent staining in RFL-6 cells incubated with EC antibody. B) After incubation with cycloheximide to stop protein synthesis, the immunolocalization is still concentrated in the Golgi complex. (Internal scale bar $=10 \mu \mathrm{m}$.)

tein, identified by the EC antibody, corresponds to the molecular weight of Goi-3 $[12,13]$. The exact mechanism of topographic targeting of $\mathrm{G}$ protein subunits to different intracellular domains, however, remains largely unknown [14].

Extracellular signals, such as hormones and growth factors, all utilize signal transduction across the cell surface to communicate with an intracellular effector [8]. This process is vital to the existence of the cell-cell differentiation and the development of an organ. Heterotrimeric $G$ proteins are classically involved in ion channel-regulation and receptor-effector coupling across cell membranes. There is evidence that Goi-3 may be a regulator of Golgi trafficking and constitutive protein secretion $[9,10]$.

Another possible function of $\mathrm{G} \alpha \mathrm{i}-3$ is the regulation of Golgi breakdown and reassembly during mitosis. This process ensures partitioning of the Golgi complex into each daughter cell. One study has followed the spatial distribution of Golgi membranes in the early stages of embryogenesis [15]. The identification of the amounts 


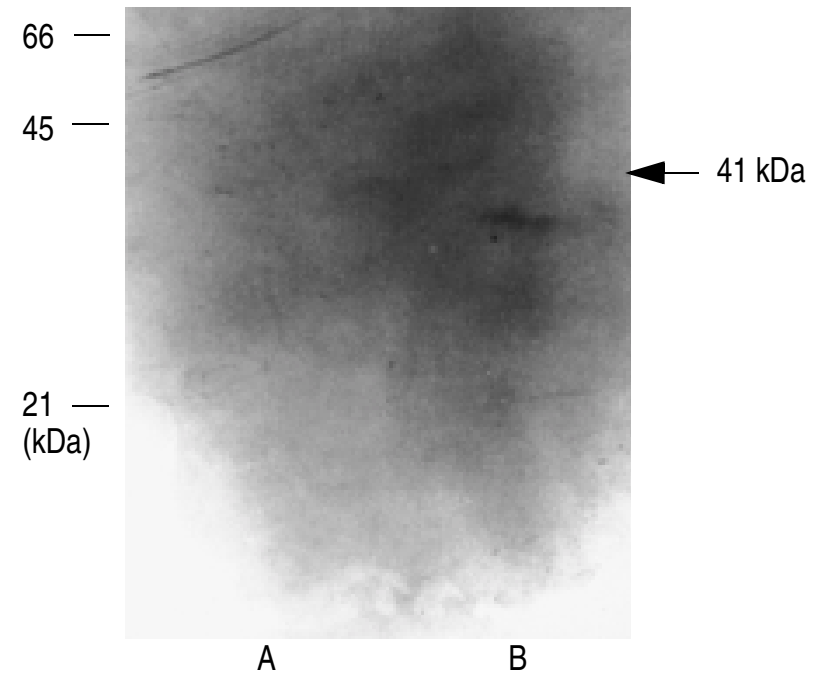

Fig. 4. - Western blot analysis of RFL-6 cellular membranes and cytosol. Each lane was loaded with equal amounts of cytosolic (A) and membrane (B) protein. There is a distinct band of $41 \mathrm{kDa}$ in lane $\mathrm{B}$ after incubation with EC antibody, which corresponds to the Gai-3 subunit.

and functions of the many $\mathrm{G}$ proteins present during cell differentiation and organ development is a future challenge. Further understanding of how the $\alpha i$ gene is controll- ed, possible alternative splicing of Gai-3 messenger ribonucleic acid and how individual subunits come to have distinct intracellular locations will advance the understan-ding of the role of $\mathrm{G}$ proteins during development.

Other studies have localized heterotrimeric $G$ proteins to the Golgi complex in mature specialized cells [9, 10], but the localization in an undifferentiated foetal lung cell line had not been previously demonstrated. The role of the heterotrimeric $\mathrm{G}$ proteins in the regulation of protein secretion in the developing lung will be the subject of future study. This study establishes the presence of Goi-3 on the Golgi, which represents a novel intracellular location for a heterotrimeric $\mathrm{G}$ protein in a nonpolar foetal cell line. Its localization further suggests many possible regulatory roles in the development of the normal lung.

Acknowledgement: The author thanks J. Zhu for technical support.

\section{References}

1. Audigier Y, Nigam N, Blobel G. Identification of a G protein in rough endoplasmic reticulum of canine pancreas. $J$
Biol Chem 1988; 263: 16352-16357.

2. Ausiello D, Holtzman E, Gronich J, Ercolani L. Cell signaling. In: Seldon DW, Griebisch G, eds. The Kidney. New York, Raven Press, 1992; pp. 645-705.

3. Bourne HR, Stryer L. G proteins: the target sets the tempo. Nature 1992; 358: 541-543.

4. Bourne H. Trimeric G proteins. Nature 1995; 270: $933-$ 934.

5. Charrier P, Parton RG, Hauri RP, Simons K, Zerial M. Localization of low molecular weight GTP-binding proteins to exocytic and endocytic compartments. Cell 1990; 62: 317-329.

6. Roa M, Cornet V, Yang CZ, Goud B. The small GTP binding protein rab6p is distributed in the cytosol by brefeldin A. J Cell Science 1993; 106: 789-802.

7. Denker S, McCaffrey JM, Palade GE, Insel P, Farquhar M. Differential distribution of alpha subunits and beta gamma subunits of heterotrimeric $\mathrm{G}$ proteins on Golgi membranes of exocrine pancreas. J Cell Biol 1996; 133 : 1027-1040.

8. Murray-Whelan R, Schlegel W. Brain somatostatin receptor-G protein interation. $\mathrm{G}$ alpha $\mathrm{C}$ terminal antibodies demonstrate coupling of the soluble receptor with $\mathrm{Gi}$ (1-3) but not with Go. J Biol Chem 1992; 267: 2960-2965.

9. Arnold HH, Salminen R. Differentiation of BA-HAN-1C rhabdomyosarcoma cells is controlled by a pertussis toxin sensitive signaling pathway. Cell Molec Biol Res 1993; 39: 195-208.

10. Bourne H, Sanders DA, McCormick F. The GTPase superfamily: a conserved switch for diverse cell functions. Nature 1990; 348: 125-132.

11. Stow JL, De Almeida B, Narula N, Holtzman E, Ercolani L, Ausiello D. A heterotrimeric G protein, Goi-3 on Golgi regulates the secretion of a heparan sulfate proteoglycan in LLCPK1 epithelial cells. J Cell Biol 1991; 114: 11131124.

12. Skorecki K, Verkman A, Ausiello DA. Cross talk between stimulatory and inhibitory guanine $5^{\prime}$ triphosphate binding proteins: a role in activation and desensitization of the adenylate cyclase response to vasopressin. Biochem 1987; 26: 639-647.

13. Speigel AM, Shenkar R, Weinstein LS. Receptor-effector coupling by $\mathrm{G}$ proteins: implications for normal and abnormal signal transduction. Endocrinol Rev 1992; 13: 536-565.

14. Stow JL. Regulation of vesicular transport by GTP binding proteins. Curr Opin Nephrol Hyper 1995; 4: 421-425.

15. Ripoche J, Link B, Yucel J, Tokuyasu K, Malhotra V. Location of Golgi membranes with reference to dividing nuclei in syncytial drosophila embryos. Proc Natl Acad Sci USA 1994; 191: 1878-1882. 